\title{
INVERSE RELATIONSHIP BETWEEN TRANSMITTER RELEASE AND TERMINAL LENGTH IN SYNAPSES ON FROG MUSCLE FIBERS OF UNIFORM INPUT RESISTANCE ${ }^{1}$
}

\author{
BRUCE M. NUDELL ${ }^{2}$ AND ALAN D. GRINNELL \\ Departments of Biology and Physiology, Jerry Lewis Neuromuscular Research Center, Ahmanson Laboratory for \\ Neurobiology, UCLA, Los Angeles, California 90024
}

Received August 6, 1981; Revised October 16, 1981; Accepted October 16, 1981

\begin{abstract}
Transmitter release at frog neuromuscular junctions is known to be related positively to nerve terminal length. However, the correlation is inexact, with a wide scatter of data. We have analyzed the endplate potentials (EPPs) of identified frog cutaneous pectoris muscle fibers, correlating release with terminal size and fiber input resistance $\left(R_{\text {in }}\right)$. Transmitter release was assayed by quantal analysis of endplate activity in low $\mathrm{Ca}^{2+}$ Ringer solution and by measurement of the EPPs evoked in normal Ringer solution and curare.

For fibers of approximately the same $R_{\text {in }}$, there is an inverse relationship between the level of transmitter release per unit length and total terminal length. Terminals with high levels of release per unit length tend to be shorter than do those which release relatively less transmitter per unit length. Furthermore, if the analysis is restricted similarly to fibers with nearly identical $R_{\text {in }}$, the total transmitter release of the largest endplates is usually less than that of the shorter terminals in the sample.

These findings do not contradict the overall trend of greater release and longer terminals on larger muscle fibers (with lower $R_{\text {in }}$ ). Instead, they help explain the variability in measurement of release versus terminal length. The relationship that we find is consistent with the hypothesis that, in the cutaneous pectoris, terminals are induced to grow until an adequate safety factor for transmission is achieved.
\end{abstract}

In a number of neuromuscular preparations, it has been noted that conditions that diminish synaptic effectiveness or cause neuromuscular inactivity result in nerve terminal growth. Thus, large diameter muscle fibers (low input resistance) tend to have larger terminals than do small diameter fibers (high input resistance) (Harris, 1954; Coërs, 1955; Coërs and Woolf, 1959; Anzenbacher and Zenker, 1963; Granbacher, 1971; Kuno et al., 1971; Korneliussen and Waerhaug, 1973; Bennett and Pettigrew, 1975; Harris and Ribchester, 1979); wintering frogs

\footnotetext{
${ }^{1}$ B. N. was supported by National Research Service Award Predoctoral Fellowship MH-IT32 1S345-03. This work was supported by grants from the United States Public Health Service (NS 06232) and a center grant from the Muscular Dystrophy Association. We wish to thank Drs. A. A. Herrera, S. Hagiwara, M. S. Letinsky, P. O'Lague, and Mr. L. O. Trussell for helpful discussions during the research and preparation of this paper. We also thank Frances Knight and Don Simpson for technical assistance.

${ }^{2}$ To whom correspondence should be addressed at the Jerry Lewis Neuromuscular Research Center, UCLA School of Medicine, Los Angeles, CA 90024.
}

display a higher incidence of sprouting than do summer frogs (Wernig et al., 1980a, b; Herrera and Grinnell, 1981); and experimental alteration of neuromuscular activity, either by presynaptic or postsynaptic block, has been shown to induce sprouting (see reviews by Brown et al., 1981; Grinnell and Herrera, 1981). Since transmitter release is correlated positively with nerve terminal size (Kuno et al., 1971; Bennett and Raftos, 1977; AngautPetit and Mallart, 1979; Harris and Ribchester, 1979; Grinnell and Herrera, 1980), nerve terminal growth is generally regarded as one of the mechanisms designed to maintain or restore an adequate safety factor for transmission.

Recent work from our laboratory has shown that synaptic efficacy at frog neuromuscular junctions can be regulated independently of changes in nerve terminal size or apparent structure. Transmitter release per unit length of terminal increases sharply when a motoneuron's peripheral arborization is reduced in size (Herrera and Grinnell, 1980) or when the contralateral homologous muscle is denervated (Herrera and Grinnell, 1981). Moreover, normal frog motor nerve terminals of equivalent 
size may differ by 10-fold or more in the amount of transmitter released (Grinnell and Herrera, 1980; present data).

The relationship between transmitter release and terminal size is, in fact, not a very precise one. The correlation coefficient of the line of best fit for frog sartorius muscle junctions was 0.43 in the findings of Kuno et al. (1971) and a similar value has been found in a large number of measurements in our laboratory (A. A. Herrera and A. D. Grinnell, unpublished observations). Accordingly, we have sought to determine whether correlation of release properties with both the morphology of identified junctions and the muscle fiber input resistance would permit a better understanding of the relationship between junctional strength and size in frog muscle. In this paper, we provide evidence that, on muscle fibers of approximately the same input resistance in the frog cutaneous pectoris (c.p.) muscle, there is an inverse relationship between terminal length and release per unit terminal length that helps identify the source of some of the variability seen in the relationship between junctional size and strength. These findings suggest a regulation of synaptic size and efficacy that works in the direction of making all junctions roughly equivalent in effectiveness.

\section{Materials and Methods}

Electrophysiology. Cutaneous pectoris muscles, dissected from pithed Rana pipiens ( 6 to $7 \mathrm{~cm}$, body length), were stretched to about $110 \%$ of the normal rest length in the animal and pinned to a thin layer of Sylgard in a Petri dish that served as a recording chamber. In studies of synaptic efficacy in frogs, it is important to control the degree of stretch since transmitter release is sensitive to this variable (Fatt and Katz, 1952; Hutter and Trautwein, 1956; Turkanis, 1973; Grinnell and Herrera, 1980). For the eight muscles in which endplate physiology and morphology were compared, the average sarcomere spacing was $2.46 \mu \mathrm{m}$, and within each muscle sarcomere, spacing usually varied by less than $5 \%$. Muscle fibers were impaled with glass micropipettes filled with $3 \mathbf{~ м ~} \mathrm{KCl}$ (20 to 50 megohms). To measure input resistance, a second micropipette, for current injection, was inserted in the same fiber at less than $50 \mu \mathrm{m}$ separation. Fibers with resting potentials less than $-80 \mathrm{mV}$ were rejected as were fibers in which either penetration was not abrupt and stable. It is to be expected that data from smaller fibers (higher input resistance) would be especially sensitive to the shunt resistance introduced by damage caused by the second electrode penetration. This was observed occasionally. However, maintenance of the $-80-\mathrm{mV}$ resting potential criterion eliminated cases of severe fiber damage, and since most of the findings described in this paper are based on a comparison of synaptic strength on fibers of the same input resistance, the effects of damage should have affected all data within a given input resistance category approximately equally. Moreover, in these experiments, we recorded endplate potential (EPP) and miniature endplate potential (mEPP) amplitudes before the second electrode penetration and measurement of input resistance. The fact that mean $\mathrm{mEPP}$ amplitude recorded in this way showed a close linear correlation with input resistance throughout the range of fiber sizes studied (see below) implies that data from high resistance fibers were not affected seriously by electrode damage.

Synaptic strength was measured under three conditions. (1) Quantal contents were determined in a Ringer solution containing $0.2 \mathrm{mM} \mathrm{CaCl}, 1 \mathrm{~mm} \mathrm{MgCl}, 116 \mathrm{mM}$ $\mathrm{NaCl}, 2 \mathrm{~mm} \mathrm{KCl}$ and buffered to $\mathrm{pH} 7.2$ with approximately $1 \mathrm{~mm} \mathrm{NaHCO}_{3}$. A minimum of 120 endplate potentials were evoked at $1 \mathrm{~Hz}$, and quantal contents were calculated using the direct method $(m=$ $\overline{\mathrm{EPP}} / \mathrm{m} \overline{\mathrm{EPP}})$. When release was reduced sufficiently, the method of failures ( $m=\ln$ (No. trials/No. failures)) was used also, yielding results that were usually in close agreement. (2) The average amplitude of 40 consecutive EPPs evoked at $1 \mathrm{~Hz}$ was determined in a Ringer solution similar to that described above but containing $1.8 \mathrm{mM}$ $\mathrm{CaCl}_{2}$, and 0.9 to $1.1 \times 10^{-5} \mathrm{M} d$-tubocurarine chloride (dTC, Nutritional Biochemicals). Even at $1 \mathrm{~Hz}$ stimulation, there is some synaptic depression. In these experiments, a 5-min rest period was allowed before recording from each endplate to ensure recovery from any depression due to prior stimulation. In all EPP measurements, synaptic potentials were normalized to a standard resting potential of $-90 \mathrm{mV}$ and corrected for nonlinear summation by the methods of Katz and Thesleff (1957) and Martin (1955), respectively. This method of calculation of release tends to overcorrect for nonlinear summation at EPP amplitudes exceeding 10 to $15 \mathrm{mV}$ (McLachlan and Martin, 1981). This source of systematic error probably did not affect our findings seriously, however, since, in most experiments, the raw EPP amplitudes did not exceed $10 \mathrm{mV}$. (The range of EPP amplitudes in the experiment of Fig. 1, for example, was 1.5 to $3.6 \mathrm{mV}$, and for the other four normal $\left[\mathrm{Ca}^{2+}\right]$ experiments of Table I, the ranges were 0.46 to $2.0,2.9$ to $9.7,3$ to 17 , and 4 to $15 \mathrm{mV}$.) In the normal $\left[\mathrm{Ca}^{2+}\right]$ experiments, EPP amplitudes also were normalized for muscle fiber input resistance. Each EPP was multiplied by the ratio of the average input resistance of all fibers sampled to the input resistance of the fiber in question. We feel that this corrected value provides a reasonably accurate measure of the relative amount of transmitter released. We chose this form of analysis rather than the method of variations (Martin, 1955) because of uncertainties in the value of the binomial parameter $n$ and in the accuracy of the correction for nonlinear summation and because of the complicating effect of synaptic depression. (3) The amplitudes of EPPs evoked by $40 \mathrm{~Hz}$ tetanic stimulation were determined in normal frog Ringer solution (as in condition 2 but without $\mathrm{MgCl}_{2}$ ) containing 1 to $2 \times 10^{-5}$ M dTC. A 15-min rest interval was allowed between the study of successive endplates. This was found to be sufficient to avoid any effects of synaptic depression surviving from the study of the previous cell. EPP amplitudes were normalized to a standard resting potential of $-90 \mathrm{mV}$ and corrected for nonlinear summation.

A potential source of error in estimating transmitter release on the basis of normalized EPP amplitude is the disparity in endplate dimensions. For a recording site near the middle of an endplate, synaptic currents arising at the ends of long junctions will contribute less to the recorded potential than equivalent currents at the ends of shorter junctions. This problem was minimized by recording from the site that displayed the shortest EPP 
rise time at any given junction. Furthermore, a plot of mean mEPP size versus input resistance in 17 fibers of one muscle showed a close correlation between these variables (linear regression correlation coefficient $=0.95$ ), with no deviation from linearity for the low input resistance fibers with the largest terminals. For these reasons, we do not feel that differences in terminal length seriously compromise our assessment of the relative levels of transmitter release. In low $\left[\mathrm{Ca}^{2+}\right]$ Ringer solution, quantal contents determined for even the longest terminals showed good agreement between the method of failures and the direct method, indicating that ambiguities in quantal size did not alter our determination of synaptic strength significantly.

In all of these experiments, the bath temperature was maintained between 11 and $13^{\circ} \mathrm{C}$.

Junctional morphology. Nerve terminals were stained by a nitro blue tetrazolium (NBT) method (Letinsky and DeCino, 1980) which shows clearly the entire terminal arborization, including fine sprouts, and by a cholinesterase (AChF) stain (Karnovsky, 1964). Muscles were fixed and stained in their recording dishes at the length studied physiologically. Following postfixation, the fibers from which recordings had been made were identified with the aid of (1) the detailed maps made during the experiment; (2) the sites of electrode penetration, which were stained darkly by NBT; and (.3) the presence of deliberately damaged adjacent fibers. In most cases, to aid in the visualization of endplates, the junctional region of the fiber was dissected out of the muscle before camera lucida drawings were made. Terminal size was measured as the summed length of NBT-stained nerve branches lying within synaptic gutters outlined by the AChE stain (see Fig. 3). Terminal length was normalized to a constant sarcomere spacing within each experiment. We very rarely saw NBT-stained terminal sprouts extending beyond AChE-filled gutters. When we did observe them, they comprised an insignificant fraction of the terminal area and therefore were not included in measurements of terminal size.

We routinely measured terminal length rather than area because of the difficulty of making light microscopic assessments of terminal width. Nevertheless, during the course of these experiments, we saw no obvious relationship between terminal length and the average width of terminal branches. As a control, 24 terminals from one muscle were drawn carefully at $\times 1200$ under oil immersion (using a Zeiss planapo $\times 100$ objective) and their areas and lengths were compared. A comparison of terminals on fibers of similar and disparate diameter failed to reveal any consistent relationship between terminal length and average terminal branch width. A plot of terminal area versus terminal length revealed a direct proportionality with little scatter (linear regression correlation coefficient $=0.97$ ). The observed mean terminal width $(1.42 \pm 0.18 \mu \mathrm{m})$ is consistent with measurements of the mean width of synaptic apposition $(1.52 \pm 0.04$ $\mu \mathrm{m})$ determined earlier by electron microscopic observation (Grinnell and Herrera, 1980). We feel that, in the absence of extensive ultrastructural information for each terminal studied, terminal length is a satisfactory measure of synaptic size.
Transmitter release per unit length. Values for transmitter release per unit terminal length were calculated by dividing the average corrected EPP size or quantal content by the junctional length. It should be pointed out that this method of analysis is insensitive to the existence of heterogeneities in release along the length of the terminal. Extracellular recordings of evoked quantal release in normal or intermediate $\mathrm{Ca}^{2+}$ concentrations support the view that release is relatively uniform (del Castillo and Katz, 1956; Katz and Miledi, 1965, 1968). However, Bennett and Lavidis (1979) have reported that, in low $\mathrm{Ca}^{2+}$ concentrations comparable to the low $\left[\mathrm{Ca}^{2+}\right]$ Ringer solution used in the present experiments, release can be highly nonuniform, with 3 to $5 \%$ of a terminal responsible for as much as 30 to $50 \%$ of the evoked response. Even if this were the case, it would simply mean that, in junctions where we see greater release per unit length, there are more, or more effective, release sites correlated with a given length of terminal, independent of the spatial distribution. In any case, the relationship between release per unit length and length described below is equally convincing at both low and normal $\mathrm{Ca}^{2+}$ concentrations.

Grouping of the data. Synaptic strength was assessed at normal $\left[\mathrm{Ca}^{2+}\right]$ in 60 fibers (five curarized muscles) and at low quantal content in 42 fibers (three muscles in 0.2 $\mathrm{mM} \mathrm{Ca}{ }^{2+}$ ). A major aim of this study was to discern more clearly the relationship between synaptic effectiveness and terminal size. The efficacy of neuromuscular junctions depends both on the amount of transmitter released and the postsynaptic input resistance. Previous studies have demonstrated that synaptic size and fiber diameter are correlated positively (see the introduction). Therefore, in order to study the interdependence of synaptic size and presynaptic release properties, we felt that it would be advantageous to analyze the relationship between these variables under conditions where postsynaptic input resistance did not vary significantly. To this end, the fibers studied in each muscle were subdivided into 3 to 5 different groups, within each of which the input resistance usually differed by less than 0.1 megohm. In most cases, it was possible to do this while taking advantage of gaps in the distribution of input resistances in the sampled population. In other cases, where no such gaps existed over a range of 0.2 megohm or more, separate groups were formed arbitrarily. In these cases, a survey of alternate groupings showed none that contradicted the conclusions presented below. Data from different muscles were not pooled or plotted together because of the different degrees of synaptic block and individual differences in absolute release levels that would have added much variability to the analysis.

\section{Results}

Figure $1 \Lambda$ shows a representative plot of transmitter release (EPP amplitude corrected for input resistance, resting potential, and nonlinear summation) at $14 \mathrm{syn}$ apses in one muscle as a function of terminal length. There is a clear tendency for the larger synapses to show larger EPPs, indicative of more transmitter release, but the correlation coefficient of the regression line is only 0.4 , and at any given terminal length, there is wide 

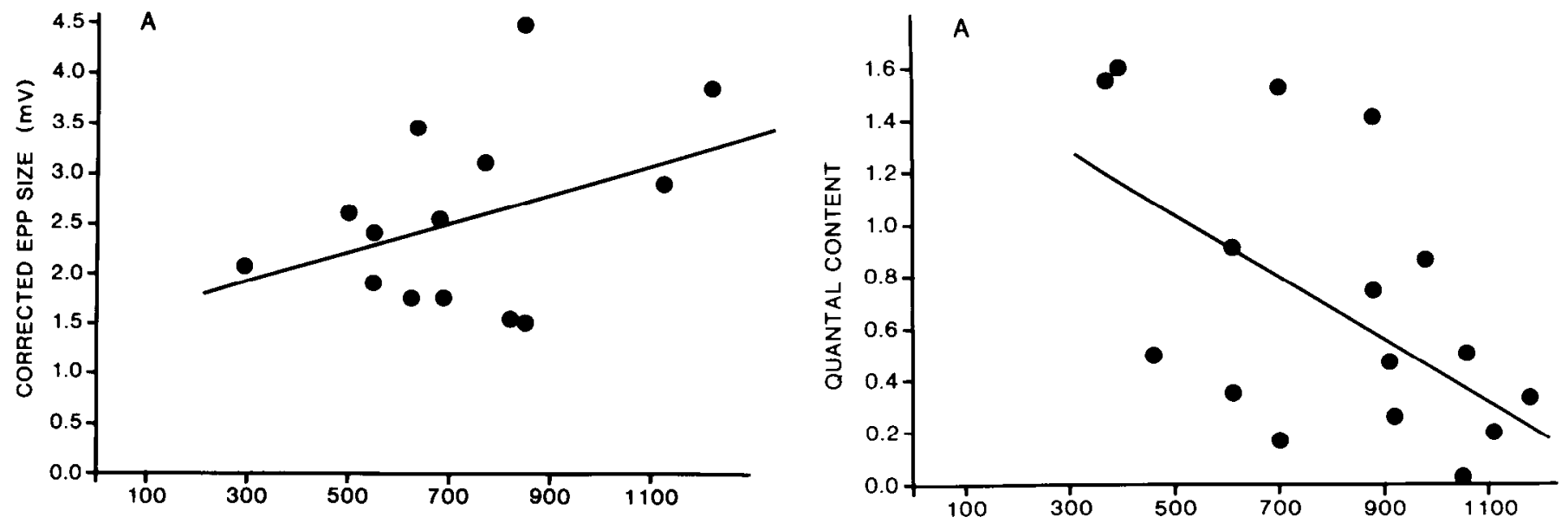

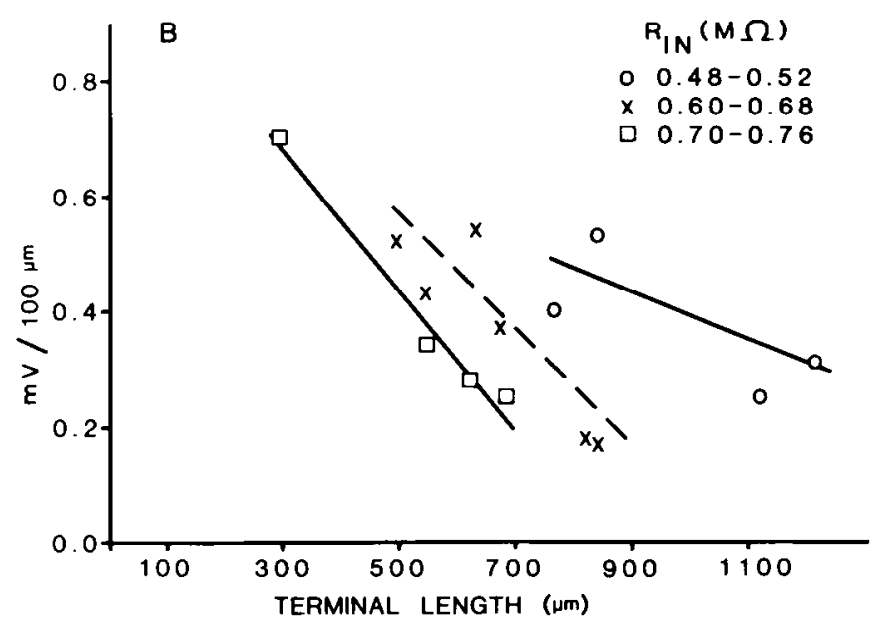

Figure 1. A, Transmitter release, judged by EPP size corrected for resting potential, nonlinear summation, and input resistance, versus terminal length for 14 endplates in one curarized c.p. muscle. The correlation coefficient for the regression line is 0.4. $B$, Data from the cells in $A$ plotted as release per unit terminal length (corrected millivolts per $100 \mu \mathrm{m}$ ) versus terminal length. Endplates are grouped on the basis of muscle fiber input resistance. The lines represent the lines of best fit.

variability in the amount of transmitter released. Of the four other experiments conducted in curare solutions, two showed positive slopes with correlation coefficients of 0.66 (14 junctions) and 0.29 (6 junctions), and two had negative slopes with correlation coefficients of 0.61 (18 junctions) and 0.87 (8 junctions). Variability was even greater when synaptic strength was assessed in low $\left[\mathrm{Ca}^{2+}\right]$ Ringer solution. Figure $2 A$ shows a sample plot of quantal content against terminal length for 16 junctions in another muscle. This plot is characteristic in that there is no convincing correlation between the two variables. In fact, the data of Figure $2 A$ show a negative slope with a correlation coefficient of 0.56 . Two other experiments in low $\left[\mathrm{Ca}^{2+}\right]$ Ringer solution revealed negative slopes, with poor correlation coefficients of 0.04 (18 junctions) and 0.31 (9 junctions). Even considering the relatively small sample sizes in several of our experiments, we were struck by the lack of correlation between terminal size and release in view of the generally accepted belief that

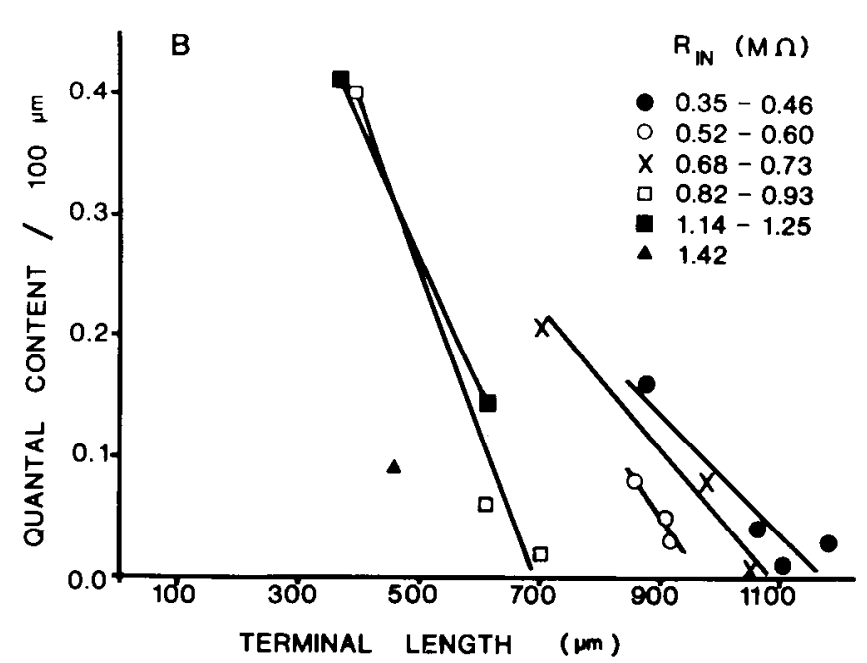

Figure 2. A, Quantal content versus terminal length for 16 endplates in one muscle. The correlation coefficient for the regression line is 0.56 . The Ringer solution contained $0.2 \mathrm{~mm}$ $\mathrm{Ca}^{2+}$ and $1 \mathrm{mM} \mathrm{Mg}^{2+} . B$, Quantal content per unit terminal length versus terminal length for the endplates in $A$. The junctions are grouped on the basis of muscle fiber input resistance. The lines represent the lines of best fit.

release is proportional to terminal size. We were surprised initially, too, by the frequent observation that, in subgroups of fibers having approximately the same input resistance, the strongest terminals (those having the largest EPPs or highest quantal contents) were often the smallest, with much higher levels of release per unit length. Hence, we made a more systematic study of the phenomenon. Figures $1 B$ and $2 B$ show the same data as in Figures $1 A$ and $2 A$ but plotted as release per unit length versus terminal length and subdivided into several different populations, each having the same input resistance within a range of approximately 0.1 megohm. Twenty-six of the 27 groups contained synapses of different release per unit length. All of these showed a clearcut inverse relationship between release per unit length and length. Moreover, as input resistance decreased, there was a shift of successive groups to the right, i.e., toward longer terminal lengths for the same level of release per unit length. There was also a marked tend- 
ency for groups having low input resistance (large fibers) to show a shallower slope for the relationship; i.e., there were larger differences in length associated with a given mean difference in transmitter release per unit length, and characteristically, the range of release per unit length values was less than in groups of smaller fibers.

Sample data are shown in Figure 3, which contains tracings of the nerve terminal morphology and of evoked release in two junctions which had input resistances of 0.91 and 0.93 megohm (data from these cells also appears in Fig. 2). Note that the longer terminal had a quantal content $(m)$ only about one-tenth as large as that of the shorter terminal.

In general, it is clear that there is no simple quantitative reciprocal relationship between terminal length and release per unit length; e.g., the ratio of values of release per unit length of two different terminals was not equal to the inverse ratio of their lengths. The differences in release per unit length were usually greater than could be explained by the differences in length. Table I compares the ralio of the highest to lowest values of release per unit length for the 26 groups studied, with the ratio of corresponding terminal lengths taken from the lines of best fit, such as those shown in Figures $1 B$ and $2 B$. The 27 th group consisted of 3 fibers in low $\left[\mathrm{Ca}^{2+}\right\rceil$ Ringer solution which varied in length by $34 \%$ and showed no consistent pattern in release per unit length. Of the 26 groups included, those in dTC and normal $\mathrm{Ca}^{2+}$ levels showed a mean $61 \%$ lower release per unit length associated with $57 \%$ longer terminals. Those in low $\left[\mathrm{Ca}^{2+}\right]$ Ringer solution showed a 79\% lower release rate associated with $45 \%$ longer terminals (see Table I).

Calculations based on the data summarized in Table I show that, if the analysis is restricted to fibers of essentially the same input resistance, the total transmitter release of the largest endplates was, on the average, $53 \%$ (in $1.8 \mathrm{mM} \mathrm{Ca}^{2+}$ ) and $28 \%$ (in $0.2 \mathrm{mM} \mathrm{Ca}^{2+}$ ) of that in the shortest terminals in the group. In support of these mean

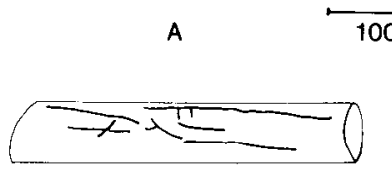

$L=395 \mu \mathrm{m}$

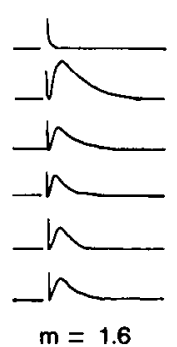

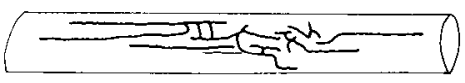

$\mathrm{L}=700 \mathrm{um}$

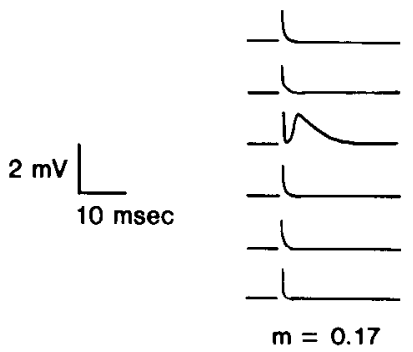

Figure 3. Tracings of the NB'T-stained terminals lying within cholinesterase-outlined gutters (not shown) which comprised the endplates on two fibers of $0.91(A)$ and 0.93 megohm $(B)$ input resistance. Also shown are tracings of 6 consecutive EPPs (out of 120) recorded at each of these junctions. The quantal content $(m=1.6)$ of the shorter junction (length $=395 \mu \mathrm{m})$ was almost 10 times that $(m=0.17)$ of the longer junction (length $=700 \mu \mathrm{m})$ values derived from the lines of best fit for each group, we found that, in 24 of the 26 groups, the EPP or quantal content of the shortest terminal was greater than that of the longest terminal. Furthermore, in each of the 26 groups, the endplate which produced the largest EPP, or released the most quanta, was shorter than the weakest junction in the group. The mean ratio of the largest to smallest EPP for the 15 groups bathed in curare solutions was $2.09 \pm 0.8$. The mean ratio of the terminal lengths of the strongest to weakest synapses was $0.69 \pm 0.16$. The corresponding values for 11 groups bathed in low $\left[\mathrm{Ca}^{2+}\right]$ Kinger solution were $8.5 \pm 14$ (mean high to low quantal content) and $0.72 \pm 0.15$ (mean strongest to weakest junctional lengths).

Although the smallest junctions within each input resistance group were absolutely stronger than the larger ones, the effect of the relationship described was to equalize their effectiveness. It is perhaps because of this apparent regulation that, at normal $\mathrm{Ca}^{2+}$ levels, all junctions in the c.p., whether large or small, on fibers of high or low input resistance, are suprathreshold to a single stimulus (Grinnell and Herrera, 1980). The differences in EPP size at $1 \mathrm{~Hz}$, therefore, are apparently functionally irrelevant. On the other hand, we do not know the natural firing frequency of the c.p. nerve, and it is possible that, at sustained higher frequencies, differences in synaptic strength might become important. To test this possibility, randomly selected junctions of different efficacy were studied at $40 \mathrm{~Hz}$, a frequency which yields maximum isometric tetanus tension in the c.p. It is apparent that, at high stimulus frequencies, other mechanisms contribute to reduce the differences in synaptic efficacy further. As Figure 4 shows, junctions with small EPPs facilitate more and remain in a potentiated state for longer periods than do initially stronger junctions. Although the strongest junctions tend to remain strongest, 2-fold differences in initial EPP size often are equalized after 2 to $2.5 \mathrm{sec}$ of $40 \mathrm{~Hz}$ stimulation. Tension measurements in normal Ringer solution show that there is virtually no decline in tension during such a $40-\mathrm{Hz}$ tetanus. Apparently the enhanced potentiation of weak junctions, coupled with the adjustment of terminal length and/or release per unit length described above, ensures that all junctions continue to remain suprathreshold even with sustained high frequency stimulation.

\section{Discussion}

Randomly selected neuromuscular junctions in the frog cutaneous pectoris (c.p.) muscle differ over a wide range in both nerve terminal length and EPP amplitude. Terminals of the same length may differ by as much as 3 (in normal $\left[\mathrm{Ca}^{2+}\right]$ )- to 10 (in $0.2 \mathrm{mM} \mathrm{Ca}{ }^{2+}$ )-fold in amount of transmitter released, and terminals with the same transmitter output may differ by more than $150 \%$ in length. These differences could represent completely random variability of little importance for junctions that have high safety margins in any case. However, it appears from the present data that this is not the case, that terminal length and strength are, in fact, co-regulated within relatively narrow limits. We have found that, if junctions are subdivided into groups based on the input resistance of the muscle fibers that they innervate, there is a clear-cut inverse relationship between release per 


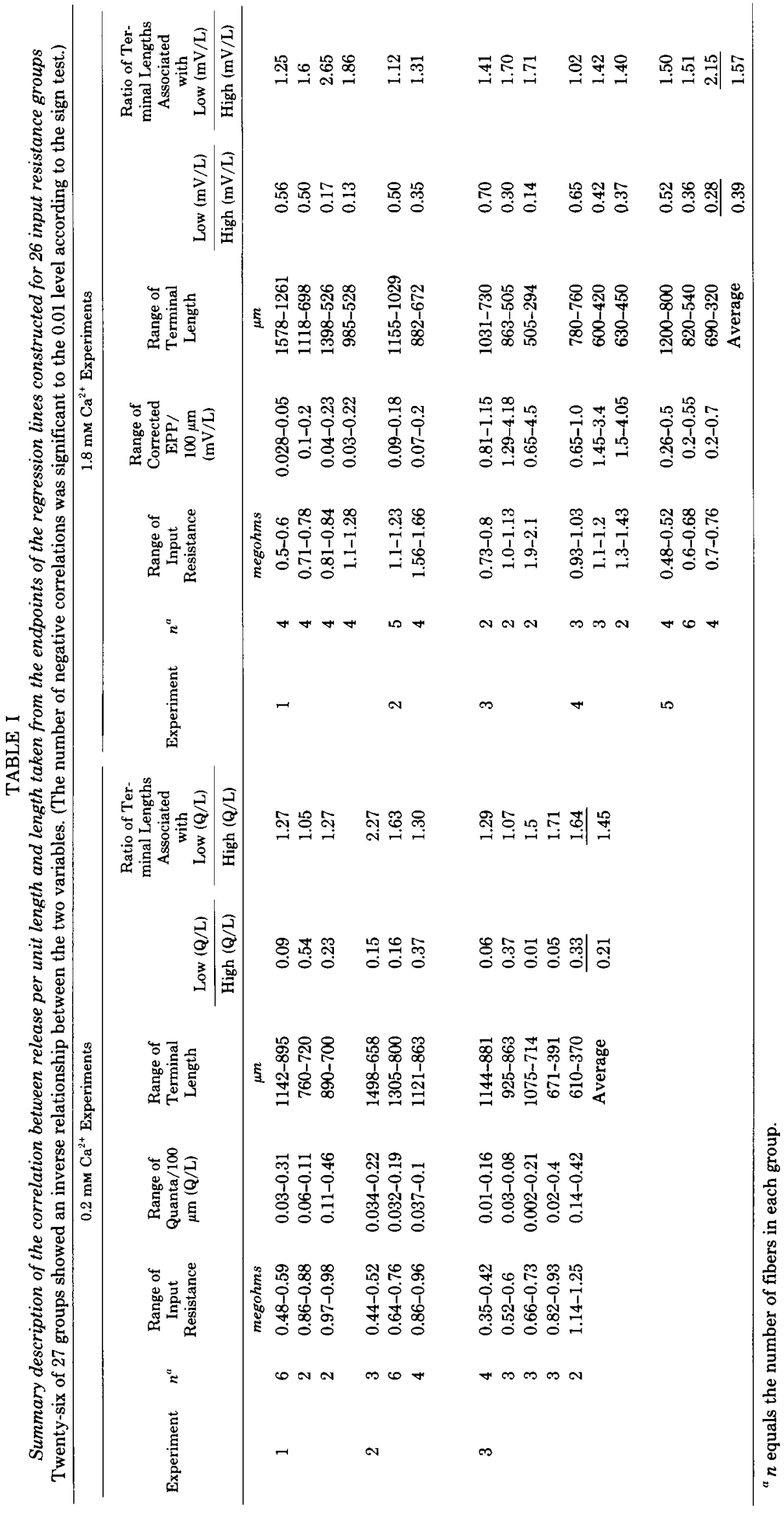




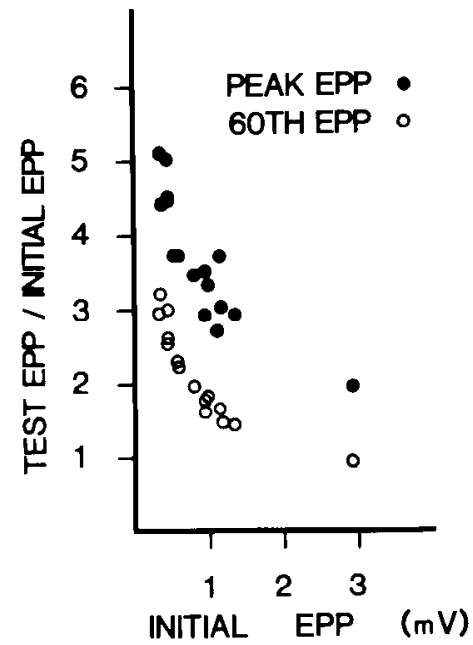

Figure 4. Ratios of the peak EPP to initial EPP and 60th EPP to initial EPP versus initial EPP for 16 endplates in one muscle during a 40-Hz, 1.5-sec train. EPPs were corrected for resting potential and nonlinear summation but not input resistance.

unit length and terminal length. In fact, short junctions within the group release so much more transmitter per unit length that almost always they are absolutely stronger than the larger junctions in the group.

While it is tempting to draw quantitative conclusions about the relationship between terminal length and release efficacy from the data presented, this would not be justified. Sample sizes are so small within each input resistance group that the range in length and release values for any given input resistance group can be considered only a partial representation of the true population. However, the overall pattern of inverse relationship between release per unit length and length appears unambiguous and provides a much better fit for the data than a simple plot of release as a function of terminal length. It is important to point out that we do not regard our data as being inconsistent with the findings of Kuno et al. (1971) and the general concept that larger fibers have larger terminals which release more transmitter. Our findings merely help explain the variability that gives rise to the poor correlation seen in the traditional relationship. In Figure $1 A$, for example, there is an overall trend toward more release from larger terminals, but there is as much or more variability at a given length (e.g., $850 \mu \mathrm{m}$ ) as there is between the mean release value for terminals of 400 and $1200 \mu \mathrm{m}$ in length. Figure $1 B$ shows that, if fiber input resistance is taken into account, the terminals of equal length but grossly different release levels belong to different input resistance groups as do terminals with the same level of release per unit length but of very different lengths.

We do not have an explanation, at a mechanistic level, for the different levels of release of weak and strong terminals, although other work in our laboratory is directed at this question. There do not appear to be differences in such obvious morphological correlates as terminal diameter (see "Materials and Methods") or spacing of active zones (B. M. Nudell and A. D. Grinnell, unpublished observations), but there are many other possible differences, both morphological and physiological, that could be involved. A clue to the nature of the mechanism underlying the observed differences may be the fact that differences in release per unit length are more dramatically manifested in low $\left[\mathrm{Ca}^{2+}\right]$ solutions. It is known that a fourth power relationship exists between external $\mathrm{Ca}^{2+}$ concentration and transmitter release at low $\mathrm{Ca}^{2+}$ concentrations and that the relationship approaches a direct proportionality at physiological levels of $\left[\mathrm{Ca}^{2+}\right]$ (Dodge and Rahamimoff, 1967). It follows, therefore, that the effect of differences in $\mathrm{Ca}^{2+}$ influx or sequestration upon transmitter release should be more pronounced in low $\left[\mathrm{Ca}^{2+}\right]$ solutions. The fact that we see such an effect is consistent with the hypothesis that at least some of the variability in release per unit length can be attributed to either enhanced $\mathrm{Ca}^{2+}$ influx or to diminished $\mathrm{Ca}^{2+}$ sequestration in strong terminals. Similar differences in $\mathrm{Ca}^{2+}$ influx or buffering are implicated in the observed differences in mean release levels of c.p. nerve terminals compared with sartorius nerve terminals (Grinnell and Herrera, 1980) and in the change in synaptic efficacy induced in sartorius junctions by altering motor unit size (Herrera and Grinnell, 1980) or by denervating the contralateral sartorius muscle (Herrera and Grinnell, 1981).

It might be argued that the observed differences in release per unit length are due to the dilution of essential components in larger terminals. This hypothesis is compatible with the finding that a decrease in terminal arborization results in an increase in synaptic strength, and it is in the right direction to explain the inverse relationship between length and release per unit length that we see. In fact, it may help to explain the reduced range in release per unit length that we frequently observe in the lowest input resistance groups. It is possible that terminals on these fibers are simply too large for motoneurons to maintain at high levels of release per unit length. However, the data indicate that a dilution model which postulates a 1:1 relationship between increment in terminal length and decrement in release per unit length could provide only a partial explanation for the observed inverse relationship. For example, the average differences in terminal length that we find on fibers of the same input resistance could, on the basis of this model, explain only $60 \%$ (high $\left[\mathrm{Ca}^{2+}\right]$ ) or $40 \%$ (low $\left[\mathrm{Ca}^{2+}\right]$ ) of the observed average difference in release per unit length (Table I). A linear dilution model could not easily account for the extreme differences in release per unit length that we occasionally see (up to 100-fold in low $\left[\mathrm{Ca}^{2+}\right]$ and 7 -fold in dTC) between terminals on fibers of equivalent input resistance. In addition, if one makes the simplifying, but we feel unlikely, assumption that, initially, all terminals are equivalent in release properties, such a model is inadequate to explain the finding that terminals on fibers of different input resistance often have the same value of release per unit length yet differ by 2 - to 3 -fold in length.

It must be emphasized that we do not know whether the regulation implied by these findings operates primarily by changes of release per unit length, by inducing continued growth of terminals that have low release levels, or both. However, if the role of the regulatory process is to achieve comparable synaptic strength on 
fibers of equivalent size, it seems likely that it is primarily length that is being adjusted, since the longest terminals remain weakest. A developmental sequence that is compatible with our results is one in which terminals differ intrinsically in release per unit length, perhaps as a function of motoneuron or motor unit size. As they form junctions on fibers of different input resistance, they grow until an adequate safety factor has been achieved. For terminals on equivalent size fibers, those that release high levels of transmitter per unit length would not be induced to grow as large as their weaker counterparts. In this manner, the differential growth of synapses with disparate release properties could help establish the apparent functional parity that we observed in the entire population. Consistent with this developmental scheme is the frequent observation that terminals innervating larger fibers showed a larger absolute difference of terminal length correlated with a given difference in release per unit length. This may be explained by the fact that, in order to achieve an equivalent increase in EPP size, keeping release per unit length constant, terminals on low input resistance fibers must grow proportionately longer than do terminals on high input resistance fibers.

The developmental scheme presented above is undoubtedly simplistic. It makes no provision for the potentially profound influence nerve may exert on muscle. For instance, it neglects the question of whether the pattern of motor unit use or differences in terminal strength are related causally to differential muscle fiber growth. It is plausible that the strongest or most frequently used synapses are, in fact, especially effective in promoting muscle fiber growth. This growth might, in turn, place added demands on the terminal, leading to a change in its length and/or effectiveness. These kinds of questions can be best answered in a developmental context. On the other hand, the inter-relationship of terminal size, release per unit length, and muscle fiber size (input resistance) compels the conclusion that these variables, in adult frogs, are closely dependent on each other. Each muscle fiber is matched to its innervation in a way that indicates that fiber size is determined by the overall effectiveness of the synapse on it or that its size determines the overall strength of that synapse. Most likely, the final state is a balance of both influences.

In the c.p., even the weakest terminals tend to be suprathreshold, perhaps because of the continued growth of the terminals with low release per unit length, driven by a regulatory mechanism such as we are proposing. In other muscles, however, such as the sartorius, where a significant fraction of the junctions are subthreshold to a single stimulus (Grinnell and Herrera, 1980), such a regulatory mechanism could be extremely important in governing the size and strength of junctions. It remains to be determined, however, whether terminals in the sartorius, with 2 or more junctions per muscle fiber, exhibit a similar inverse relationship between terminal length and release per unit length on fibers of the same input resistance.

\section{References}

Angaut-Petit, D., and A. Mallart (1979) Dual innervation of endplate sites and its consequences for neuromuscular trans- mission in muscles of adult Xenopus laevis. J. Physiol. (Lond.) 289: 203-218.

Anzenbacher, H., and W. Zenker (1963) Uber die Grossenbeziehung der Muskelfasern zu ihren motorischen Endplatten und Nerven. Z. Zellforsch. Mikrosk. Anat. 60: 860-871.

Bennett, M. R., and N. A. Lavidis (1979) The effect of calcium ions on the secretion of quanta evoked by an impulse at nerve terminal release sites. J. Gen. Physiol. 74: 429-456.

Bennett, M. R., and A. G. Pettigrew (1975) The formation of synapses in amphibian striated muscle during development. J. Physiol. (Lond.) 252: 203-239.

Bennett, M. R., and J. Raftos (1977) The formation and regression of synapses during the re-innervation of axolotl striated muscles. J. Physiol. (Lond.) 265: 261-295.

Brown, M. C., R. S. Holland, and W. G. Hopkins (1981) Motor nerve sprouting. Annu. Rev. Neurosci. 4: 17-42.

Coërs, C. (1955) Les variations structurelles normales et pathologiques de la jonction neuromusculaire. Acta Neurol. Psychiatr. Belg. 55: 741-866.

Coërs, C., and A. L. Woolf (1959) The Innervation of Muscle. A Biopsy Study, Blackwell Scientific, Oxford.

del Castillo, J., and B. Katz (1956) Localization of active spots within the neuromuscular junction of the frog. J. Physiol. (Lond.) 132: 630-649.

Dodge, F. A., and R. Rahamimoff (1967) Cooperative action of calcium ions in transmitter release at the neuromuscular junction. J. Physiol. (Lond.) 193: 419-432.

Fatt, P., and B. Katz (1952) Spontaneous subthreshold activity at motor nerve endings. J. Physiol. (Lond.) 117: 109-128.

Granbacher, N. (1971) Uber die Grossenbeiziehungen der Muskelfasern zu ihren motorischen Endplatten und nerven bei Hypertrophie und Atrophie. Z. Anat. Entwicklungsgesch. 135: 76-87.

Grinnell, A. D., and A. A. Herrera (1980) Physiological regulation of synaptic effectiveness at frog neuromuscular junctions. J. Physiol. (Lond.) 307: 301-317.

Grinnell, A. D., and A. A. Herrera (1981) Specificity and plasticity in neuromuscular connections; long term regulation of motoneuron function. Prog. Neurobiol., in press.

Harris, C. (1954) The morphology of the myoneural junction as influenced by neurotoxic drugs. Am. J. Pathol. 30: 501-519.

Harris, J. B., and R. R. Ribchester (1979) The relationship between endplate size and transmitter release in normal and dystrophic muscles of the mouse. J. Physiol. (Lond.) 296: 245-265.

Herrera, A. A., and A. D. Grinnell (1980) Transmitter release from frog motor nerve terminals depends on motor unit size. Nature 287: 649-651.

Herrera, A. A., and A. D. Grinnell (1981) Contralateral denervation causes enhanced transmitter release from frog motor nerve terminals. Nature 291: 495-497.

Hutter, O. F., and W. Trautwein (1956) Neuromuscular facilitation by stretch of motor-nerve endings. J. Physiol. (Lond.) 113: $610-625$.

Karnovsky, M. J. (1964) The localization of cholinesterase activity in rat cardiac muscle by electron microscopy. J. Cell Biol. 23: 217-232.

Katz, B., and R. Miledi (1965) Propagation of electrical activity in motor nerve terminals. Proc. R. Soc. Lond. (Biol.) 161: 453-482.

Katz, B., and R. Miledi (1968) The effect of local blockage of motor nerve terminals. J. Physiol. (Lond.) 199: 729-741.

Katz, B., and S. Thesleff (1957) On the factors which determine the amplitude of the miniature end-plate potential. J. Physiol. (Lond.) 137: 267-278.

Korneliusson, H., and O. Waerhaug (1973) Three morphological types of motor nerve terminals in the rat diaphragm and their possible innervation of different muscle fiber types. $Z$. 
Anat. Entwicklungsgesch. 140: 73-84.

Kuno, M., S. A. Turkanis, and J. N. Weakly (1971) Correlation between nerve terminal size and transmitter release at the neuromuscular junction of the frog. J. Physiol. (Lond.) 213: $545-556$.

Letinsky, M. S., and P. DeCino (1980) Histological staining of pre- and postsynaptic components of amphibian neuromuscular junctions. J. Neurocytol. 9: 305-320.

Martin, A. R. (1955) A further study of the statistical composition of the end-plate potential. J. Physiol. (Lond.) 130: 114-122.

McLachlan, E. M., and A. R. Martin (1981) Non-linear summation of end-plate potentials in the frog and mouse. J.
Physiol. (Lond.) 311: 307-324.

Turkanis, S. A. (1973) Effects of muscle stretch on transmitter release at end-plate of rat diaphragm and frog sartorius muscle. J. Physiol. (Lond.) 230: 391-403.

Wernig, A. M., M. Pécot-Dechavassine, and H. Stöver (1980a) Signs of nerve regression and sprouting in the frog neuromuscular synapse. In Ontogenesis and Functional Mechanisms of Peripheral Synapses, J. Taxi, ed., Vol. 13, pp. 225-238, Elsevier Press, Amsterdam.

Wernig, A. M., M. Pécot-Dechavassine, and H. Stöver (1980b) Sprouting and regression of the nerve at the frog neuromuscular junction in normal conditions and after prolonged paralysis with curare. J. Neurocytol. 9: 277-303. 